\title{
Non-Infectious Complications of Non-Tunneled Central Venous Catheterization for Hemodialysis: Incidence and Reasons in Ouagadougou (Burkina Faso)
}

\author{
Gérard Coulibaly*, Gildas Ilboudo, Adama Roger Karambiri, François P. Kissou, \\ Adama Lengani \\ Department of Nephrology and Hemodialysis, Yalgado Ouedraogo University Hospital Center (YO-UHC), \\ Ouagadougou, Burkina Faso \\ Email: "coulibalygerard@hotmail.fr, gildas.ilboudo@yahoo.fr, adamaroger@yahoo.fr, \\ francois@petitsamba.net, alengani2002@yahoo.fr
}

Received 21 January 2016; accepted 25 March 2016; published 28 March 2016

Copyright (C) 2016 by authors and Scientific Research Publishing Inc.

This work is licensed under the Creative Commons Attribution International License (CC BY).

http://creativecommons.org/licenses/by/4.0/

c) (i)

Open Access

\section{Abstract}

Goal: Evaluate the importance and reasons of non-infectious complications of non-tunneled central venous catheterization in our hemodialysis unit. Patients and methods: The study, a prospective type, was conducted in the department of nephrology and hemodialysis of Yalgado Ouedraogo University Hospital Center (YO-UHC) in Ouagadougou, Burkina Faso, from 15 February to 30 June 2015. Patients in whom a new central venous catheter (CVC) was inserted during the study period were included. Catheterization-related complications were noted. Results: During the study period, 156 CVCs (9 per week) were placed in femoral (56.4\%), internal jugular (40.4\%) or subclavian vein (3.2\%). There were 114 patients $(59.7 \%$ of men and $40.3 \%$ of women), average age $41.8 \pm 17.1$ years, low socio-economic level in $64 \%$ of cases. At least a non-infectious complication was observed in 67 cases representing $42.9 \%$. They were: puncture failure $(40 \%)$, arterial puncture $(12.2 \%)$, puncture of the thoracic duct $(1.3 \%)$, pneumothorax $(1.3 \%)$, bleeding related to the catheter insertion (5.8\%), hematoma $(1.3 \%)$, opposite direction $(0.6 \%)$, dysfunction of the CVC (10.3\%), femoralthrombophlebitis (3.2\%). Conclusion: Non-infectious complications of non-tunneled central venous catheterization in our hemodialysis unit were frequent and sometimes severe. Their common denominator was the absence of ultrasound guidance. Our study reaffirms the need for equipping with Doppler ultrasound in our hemodialysis units, even in de-

"Corresponding author.

How to cite this paper: Coulibaly, G., Ilboudo, G., Karambiri, A.R., Kissou, F.P. and Lengani, A. (2016) Non-Infectious Complications of Non-Tunneled Central Venous Catheterization for Hemodialysis: Incidence and Reasons in Ouagadougou (Burkina Faso). Open Journal of Nephrology, 6, 1-9. http://dx.doi.org/10.4236/ojneph.2016.61001 
veloping countries, for better security of the patient during central venous catheterization.

Keywords

Burkina Faso, Catheterization, Complication, Hemodialysis, Pneumothorax, Thrombophlebitis

\section{Introduction}

The hemodialysis session requires access to the patient's blood. This access is possible via a central venous catheter (CVC) of hemodialysis inserted into a vein of sufficient gauge, or by an arterialized vein resulting from an arteriovenous fistula (AVF) created surgically. The CVC has the advantage over the AVF to be immediately usable. The prevalence of its use in western countries varies from $7 \%$ to $39 \%$ [1]. However, the use of CVCs is more often beset by complications of various natures. The infectious complication is the most common. The non-infectious complications are not rare and can be life-threatening for patients [2]. They are probably more common in the context of lack of equipment and/or human resources such as in the sub-Saharan African countries. Burkina Faso, a developing country, currently has a single functional hemodialysis unit. The prevalence of the use of CVCs is high because of the difficulty to create the AVFs for chronic hemodialysis. The CVCs available are short-lived and yet used for long periods of time in our unit. This would contribute to increase the frequency of non-infectious complications. In this study, we wanted to assess their importance and their reasons in our hemodialysis unit.

\section{Patients and Methods}

We conducted a short cross-sectional study in the department of nephrology and hemodialysis of Yalgado Ouedraogo University Hospital Center (YO-UHC) in Ouagadougou, Burkina Faso. It covered the period from 15 February to 30 June 2015.

The hemodialysis unit was opened in 2000. Its initial capacity of nine hemodialysis stations has been extended to 29 since 2009, for about 250 patients at the time of the study. The modality used is conventional hemodialysis. Patients are admitted to the unit for chronic hemodialysis in end-stage renal disease, or acute hemodialysis. Despite the insufficient capacity of the unit, there is still no official text governing access for patients to chronic hemodialysis.

The unit benefits a state subsidy. To enter the chronic hemodialysis program, the patient must pay a lump sum of about US \$840; CVC are given free. The acute hemodialysis costs about US \$25. Drug costs and diagnostic tests are entirely the responsibility of the patient. The majority of patients are from the city of Ouagadougou.

The CVCs insertion was performed by the physicians of the department (three seniors and two new physicians) in a room dedicated to this activity, but not equipped with ultrasound. The common anatomical landmarks are used for insertion. A chest x-ray face is performed after the insertion of a CVC at the neck. Maintenance of the CVC is explained to patients. It is removed at the end of use or in the case of complication.

The CVCs used at the time of the study were short-lived, made of polyurethane and tuneless. Their length was 15 or $20 \mathrm{~cm}$. The CVC measuring $15 \mathrm{~cm}$ was preferentially inserted into the internal jugular or right subclavian veins; those measuring $20 \mathrm{~cm}$ were inserted into the internal jugular, left subclavian or femoral veins. The tunneled CVC was not yet available neither used in the unity. The unit did not have ultrasound.

The study population consisted of patients receiving chronic hemodialysis in the department of nephrology and hemodialysis unit of YO-UHC. Patients in whom a new catheter was inserted during the study period were included. We have not included cases where the CVC was changed based on recommendation.

We considered as complications the following:

- puncture failures;

- aberrant direction of the CVC;

- accidental punctures: arterial, of the thoracic duct (the puncture brings a milky liquid);

- pneumothorax: air in the pleural space. The diagnosis is suggested by a chest pain and respiratory discomfort. The diagnosis is confirmed by a chest $\mathrm{x}$-ray andthoracentesis;

- thrombophlebitis on CVC: presence of thrombus on doppler study. Dysfunction of the CVC: no or insuffi- 
cient blood flow;

- hemorrhage: unusual bleeding at the site of puncture;

- hematoma: painful swelling of the skin next to the site of puncture.

Sociodemographic, clinical and paraclinical data (the workup prior to the insertion of the CVC involved only new patients) were collected. Patients were followed from the CVC insertion to its removal.

Data were collected from the clinical examination of patients and the clinical record. A survey form was used to collect these data. The data have been processed on PC using the software Epi Info version 7.1.3.3. For the comparison of qualitative variables, Chi square and exact Fischer tests were used. The student's t test was used for comparison of quantitative variables. The level of statistical significance was set for a probability $p \leq 0.05$.

The consent of the patients (or their representative if they are unconscious) was obtained before their inclusion. They have been informed of the objectives and constraints of the study, and necessary monitoring measures. The study data were collected in the strict respect of anonymity and confidentiality. The YO-UHC lacked ethics committee at the time of the study.

\section{Results}

\subsection{General Aspects}

During the study period, 156 CVCs were placed representing approximately nine per week. These CVC insertion sites were diverse (Table 1).

114 patients were involved: 69 new patients (60.5\%) for a first session of hemodialysis (acute or chronic) and 45 former patients already in chronic hemodialysis representing 39.5\%. Among the 69 new patients, 20 patients representing $29 \%$ were in acute hemodialysis and 49 patients representing $71 \%$ in chronic hemodialysis. During the study period, 82 patients $(71.9 \%)$ received a single CVC, 25 patients $(21.9 \%)$ two CVCs, four patients (3.5\%) three CVCs and three patients $(2.7 \%)$ four CVCs.

The 114 patients included 68 men representing $59.7 \%$ and 46 women representing $40.3 \%$. The sex ratio was 1.5. The average age of the patients was $41.8 \pm 17.1$ years (extreme $=5$ and 85). It was $41.9 \pm 17.1$ years for men and $41.5 \pm 17.3$ years for women. There was no statistically significant difference for these two age groups $(\mathrm{p}=0.9)$.

Seventy-three patients (64\%) had a low socioeconomic level. The other patients had an average level (30 patients representing 26.3\%) or high (11 representing 9.7\%).

Personal history of patients is summarized in Table 2.

The total number of CVCs received before the study by 45 former patients in chronic hemodialysis was 153 representing an average of $3.4 \pm 2.3$ per patient.

When placing the CVC, 25 patients representing $16 \%$ were febrile and 30 patients representing $19.2 \%$ haddyspnea. In addition, we noted: edema of the lower limbs (62 patients representing 39.7\%), global heart failure (17 cases representing 10.7\%), right heart failure ( 4 cases representing $2.5 \%$ ), comatose state ( 2 cases representing 1.3\%). The mean arterial pressures were $148.6 \pm 24.6 \mathrm{~mm} \mathrm{Hg}($ extreme $=70-218)$ and $89.8 \pm 17.0$

Table 1. Insertion sites of central venous catheters for hemodialysis.

\begin{tabular}{lcc}
\hline & Number & Percentage \\
\hline Femoral vein & & 37.8 \\
Right & 59 & 18.6 \\
Left & 29 & $\mathbf{5 6 . 4}$ \\
\hline Total 1 & $\mathbf{8 8}$ & 34.6 \\
\hline Internal jugular vein & 54 & 5.8 \\
Right & 9 & $\mathbf{4 0 . 4}$ \\
Left & $\mathbf{6 3}$ & 2.6 \\
Total 2 & & 0.6 \\
Subclavian Vein & 4 & $\mathbf{3 . 2}$ \\
Left & 1 & $\mathbf{1 0 0}$ \\
\hline Total 3 & $\mathbf{5}$ & $\mathbf{1 5 6}$ \\
\hline Total & & \\
\hline
\end{tabular}


Table 2. Distribution of patients based on personal medical history.

\begin{tabular}{ccc}
\hline Personal Medical History & Number & Percentage \\
\hline Arterial Hypertension & 62 & 54.4 \\
Thrombophlebitis on Central Venous Catheter* & 13 & 28.9 \\
Diabetes & 9 & 7.9 \\
Gout & 6 & 5.3 \\
Human Immunodeficiency Virus Infection & 3 & 2.6 \\
AL Lambda Amyloidosis & 1 & 0.9 \\
\hline
\end{tabular}

*: The research was conducted in 45 chronic hemodialysis patients.

$\mathrm{mm} \mathrm{Hg}$ (extreme $=40$ and 125 ) respectively for the systolic and diastolic blood pressures.

The paraclinical workup prior to insertion of the CVC was done for 69 new patients. The average serum creatinine before hemodialysis was $2364.8 \pm 1073.8 \mu \mathrm{mol} / \mathrm{L}$ (Table 3 ).

\subsection{Non-Infectious Complications}

At least a non-infectious complication was observed in 67 cases representing $42.9 \%$.

\subsubsection{Puncture Failure}

The global rate of puncture failure was $40 \%$. The average number of punctures in the CVC insertion vein was $1.8 \pm 1.4$ per patient (extreme $=1$ and 8$)$. It was $1.6 \pm 1.1($ extreme $=1$ and 6$)$ for men and $2 \pm 1.7($ extreme $=1$ and 8$)$ for women. The difference between the gender was not statistically significant $(p=0.1)$. The average number of punctures of the vein did not vary significantly according to the CVC insertion site (Table 4).

One hundred-thirty-nine CVCs representing $89.1 \%$ were inserted without change of site. In the 17 remaining cases, the change of insertion site was motivated by:

- no progression of the guide: five CVCs representing 29.4\%;

- iterative arterial puncture: seven CVCs representing $41.2 \%$;

- difficulty in tracking the vein: five CVCs representing $29.4 \%$.

\subsubsection{Puncture Accidents}

An arterial puncture was observed during the insertion of 19 CVCs representing 12.2 percent of the 156 CVCs. The affected artery was the femoral (11 cases representing $12.5 \%$ of the 88 femoral CVCs) or the right carotid artery ( 8 cases representing $12.7 \%$ of the 63 internal jugular CVCs).

The thoracic duct was punctured during insertion of two CVCs in the right internal jugular vein representing $1.3 \%$ of the 156 CVCs. A pneumothorax was observed during insertion of two CVCs in the right internal jugular vein representing $1.3 \%$ of the $156 \mathrm{CVCs}$ and $3.2 \%$ of the 63 jugular CVCs.

\subsubsection{Hemorrhage}

A hemorrhage through the insertion hole of the CVC was observed after insertion of nine CVCs representing $5.8 \%$ of 156 CVCs. It concerned the femoral vein in 8 cases representing $9.1 \%$ of $88 \mathrm{CVCs}$ inserted into the femoral vein and the jugular vein in one case representing $1.6 \%$ of $63 \mathrm{CVCs}$ inserted into the internal jugular vein. A hematoma was observed when inserting two CVCs in the internal jugular vein, representing $3.2 \%$ of jugular CVCs.

\subsubsection{Insertion Path Accident}

One CVC representing $0.6 \%$ has taken the wrong direction into the right internal jugular vein while attempting to insert it into the right subclavian vein. This path has been favored by the presence of a large thrombus (as seen on the subsequent Doppler) into the right subclavian vein just downstream of the right subclavian-jugular venous confluence (Figure 1). 
Table 3. Results of laboratory tests in 69 new patients on hemodialysis.

\begin{tabular}{cccc}
\hline & $\mathbf{n}(\mathbf{\%})$ & $\mathbf{m} \pm \mathbf{S D}$ & Extreme \\
\hline Serum Creatinine $(\mu \mathrm{mol} / \mathrm{L})$ & $69(100)$ & $2364.8 \pm 1073.8$ & $444-5868$ \\
Azotemia $(\mathrm{mmol} / \mathrm{L})$ & $69(100)$ & $38.1 \pm 16.1$ & $10.4-99.8$ \\
CRP $(\mathrm{mg} / \mathrm{L})$ & $13(18.8)$ & $89.9 \pm 143.7$ & $0.2-479$ \\
Hemoglobin $(\mathrm{g} / \mathrm{dL})$ & $69(100)$ & $7.3 \pm 1.5$ & $3.7-11.7$ \\
Leukocytes (éléments $\left./ \mathrm{mm}^{3}\right)$ & $69(100)$ & $8612.8 \pm 6735.5$ & $1200-39100$ \\
Platelets (éléments $\left./ \mathrm{mm}^{3}\right)$ & $69(100)$ & $238126.1 \pm 147420.1$ & $20000-927000$ \\
\hline
\end{tabular}

CRP: C Reactiv Protein; m: mean; n: number of patients who completed the biological examination; SD: standard deviation.

Table 4. Average number of punctures according to the insertion site of the central venous catheter.

\begin{tabular}{ccc}
\hline Insertion Site of the Catheter & m \pm SD & Extreme \\
\hline Femoral Vein & $1.9 \pm 1.6$ & 1 et 8 \\
Internal Jugular vein & $1.6 \pm 1.1$ & et 5 \\
Subclavian vein & $2.6 \pm 1.5$ & 1 et 5 \\
\hline
\end{tabular}

$\mathbf{m} \pm \mathbf{S D}:$ mean \pm standard deviation; $\mathrm{p}=0.3$.

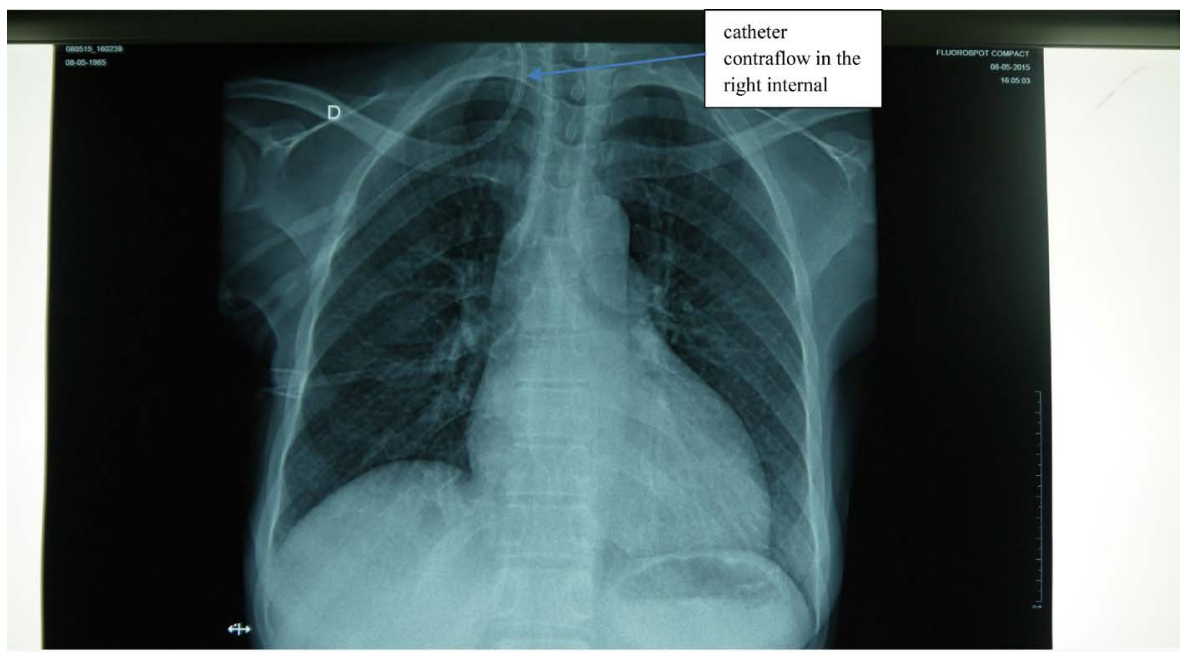

Figure 1. Aberrant course of a central venous catheter for hemodialysis inserted in the right subclavian vein, diverted contraflow in right internal jugular vein due to thrombosis of the right jugular-carotid confluence.

\subsubsection{Dysfunctions of the CVC}

The dysfunction of the CVC was observed in 16 cases representing $10.3 \%$. It involved 13 femoral CVCs representing $14.8 \%$ of femoral CVCs, three jugular CVCs representing $4.8 \%$ of internal jugular CVCs. The dysfunction was precocious in three cases and caused by an intraluminal thrombosis (one case), a wrong direction (one case); and undetermined in the third case. In 13 cases, the dysfunction was late. It concerned the CVCs inserted in femoral vein in 11 cases. It was due to an intraluminal thrombosis of the CVC (seven cases), a bending of the CVC (one case) and a thrombophlebitis on CVC (five cases).

\subsubsection{Thrombophlebitis on CVC}

There were five thrombophlebitis on CVC representing 3.2\% of $156 \mathrm{CVCs}$. The CVCs in question were all inserted into a femoral vein. Four women and one man were involved. The average duration of use of these CVCs 
was $26.8 \pm 24.1$ days (extreme $=9$ and 67) vs $26 \pm 28.4$ days (extreme $=0-122$ ) for the femoral CVCs without thrombophlebitis.

\section{Discussion}

Our study took place in a context of limited resources. As such, the means were not always available to enable good practices according to international recommendations on catheterization for hemodialysis. Nevertheless, our study has the merit of showing the reality of our hemodialysis unit; providing results that are reliable and could serve us for the improvement of our hemodialysis patients' care.

Our study population was young; the average age was $41.8 \pm 17.1$ years, as in most of the developing countries [3] [4]. In developed countries, patients are much older with an average age above 62 years [5] [6] at the initiation of the treatment by hemodialysis.

The majority of our patients had a low socioeconomic level (64\%). It fits in the context of general poverty in Burkina Faso, ranked 181 among 187 countries with a low human development index (0.388) [7]. The prevalence of diabetes of $7.9 \%$ in our sample was low compared to the results reported by some authors in developing countries where it may reach $41 \%$ [8]. Our patients, with their young age and low frequency of comorbidities such as diabetes, should in principle have a better vascular condition than those of developed countries.

The activity of CVC insertion was very important in our hemodialysis unit. In fact, about nine CVCs were inserted per week. The 45 chronic hemodialysis patients received an average three CVCs by patient. This is explained by the very frequent use of temporary CVCs due to a long delay for making AVFs or lack of possibilities to repair them. This long delay was related to the lack of surgeon availability and financial means for a significant proportion of patients.

The CDC (Center of Diseases Control) and KDOQI [9] [10] recommendations have not always been respected in the case of chronic hemodialysis. Indeed, the most used vein was the femoral vein $(50.7 \%)$ then the internal jugular vein $(45.5 \%)$ and the subclavian vein $(3.8 \%)$. However, our results are comparable to those reported by Sanogo [11] in Mali where the CVC insertion sites, in order of frequency, were the femoral veins $(53.8 \%)$ and internal jugular vein $(44.6 \%)$. In our serie, the high frequency of CVCs insertion in femoral veins for chronic hemodialysisis due to various reasons: high proportion of patients with orthopnea (19.2\%) therefore cannot withstand the supine position, lack of appropriate CVC lengths and mastery of CVC insertion into jugular veins by junior doctors at the time of the study.

Regarding acute hemodialysis, the recommendations of learned societies have been met. Thus, the femoral vein was used first (87.5\%). No CVC has been inserted into the subclavian vein. The reasons given for the cases of chronic hemodialysis patients reveal the poor care conditionsin our country: hemodialysis session every four days, lack of compliance of some patients to the salt and water restriction, bad control of pressure sometimes due to a therapeutic rupture related to lack of financial means.

The change of the site of insertion was observed with $10.9 \%$ of CVCs. The main reasons were the non-progression of the CVC guide (41.2\%), iterative arterial punctures $(29.4 \%)$ and the difficulty of locating the insertion vein $(29.5 \%)$. These reasons are mainly related to the lack of use of ultrasound guidance. Thus, one could for example avoid unnecessary punctures in the cases of stenosis and thrombosis that could have been revealed by a previous Doppler ultrasound. The lack of operator change in case of iterative puncture failures should not be overlooked. During our study, the change of operator for the same patient was only after multiple puncture failures or puncture failure after changing site. However, the risk of accidental arterial puncture is associated with the increased number of percutaneous puncture [12] [13]. Therefore, authors such as Mason and al. [14] recommend changing operator after two unsuccessful punctures, and three for Laksiri et al. [15]. The change of operator in our context is not always obvious because of the small number of the medical staff compared to the workload in the department of nephrology and hemodialysis. Thus, the difficulty of changing operator (because of reduced medical staff) and the absence of ultrasound guidance have contributed to the high rate of failures and puncture site change.

Table 5 compares mechanical complications of our series with those of other authors.

In our context, the high incidence of complications mentioned in Table 5 is directly related to the blind insertion technique of the CVC. Our department did not have an ultrasound at the time of the study. The blind insertion technique uses anatomical landmarks assumed to be fixed, which is not always the case. Indeed, the anatomical variants of internal jugular and the femoral veins are seen in more than $15 \%$ of cases [17]. The ultra- 
Table 5. Frequency of mechanical complications reported by various authors according to the insertion site of the central venous catheter.

\begin{tabular}{cccc}
\hline & Internal Jugular (\%) & Subclavian (\%) & Femoral (\%) \\
\hline Arterial Puncture & & & $9-15$ \\
McGee* & $6.3-9.4$ & $1.1-4.9$ & - \\
Iovino [16] & 12.7 & 2.7 & 12.5 \\
Our serie & & 0 & NA \\
Pneumothorax & $<0.1-0.2$ & $1.5-3.1$ & NA \\
McGee* & 0 & 3.1 & NA \\
Iovino [16] & 3.2 & 0 & $3.8-4.4$ \\
Our serie & & $1.5-3.1$ & - \\
Hematoma & $<0.1-2.2$ & - & 0 \\
McGee* & - & 0 & \\
Iovino [16] & 3.2 & & \\
Our serie & &
\end{tabular}

*: Data of several authors reported by McGee et al. [12]; NA: not applicable.

sound guidance, in agreement with the current international recommendations, is considered as the technique of reference for the insertion of CVCs [18]. The ultrasound, by guiding the puncture into the vein and detecting anatomical variations, improves the success rate and comfort, and decreases the insertion time, the number of punctures, the number of complications and the cost [19] [20]. This could also help reduce the risk of being sued to which any operator may be exposed [21].

A case of CVC in the wrong direction in the right internal jugular vein was observed. The incident occurred in a patient who had previously received several CVCs (two in the right subclavian vein and three in the right internal jugular vein). The Doppler has highlighted a large thrombus just downstream of the jugular-right subclavian confluence. Few similar cases were reported. Merrer et al. [14] in his comparative study of subclavian and femoral sites had observed $5.6 \%$ of path errors among the CVCs inserted into the subclavian vein. Such a path could seriously disrupt the cerebral hemodynamics. Once again, the lack of ultrasound guidance was a factor favoring a non-infectious complication.

In our study, CVC dysfunctions were observed in 16 cases. They involved the femoral site in 13 cases representing $81.3 \%$, but $14.8 \%$ of femoral CVCs. A prospective randomized study compared the femoral and internal jugular sites in terms of dysfunction of the CVC. It was found that the femoral and jugular sites were equivalent in terms of dysfunction of CVCs, subject to the use of a CVC adapted to the specificities of the femoral route (length $24 \mathrm{~cm}$ ). However, the left internal jugular exposes to a higher risk of dysfunction compared to the femoral and right internal jugular approach [22]. In our study, the femoral site was the mostly used, which may explain its predominance in the dysfunction frequency. If our jugular CVCs were tunneled, that would have further reduced the risk of dysfunction in jugular site [23]. So we can remember that the nature of the CVC that we used intervened in the high frequency of observed dysfunctions.

The use of a CVC very frequently leads to the formation of a fibrous sheath in the vein where the CVC is placed. The thromboses arising the reform are very often asymptomatic. For these reasons, they are undervalued. During the study of Timsit and al on 208 CVCs, the Doppler of the catheterized sites has highlighted a thrombosis in $42 \%$ of cases but it was occlusive in only $3 \%$ of cases [24]. A postmortem study on 72 patients done by Raad et al. [25] found a mural thrombosis at the level of the catheterized vein in $38 \%$ of cases and right atrial mural thrombosis in $5.6 \%$ of cases.

The incidence rate of thrombosis is variable according to the CVC insertion site. The jugular and femoral sites are more thrombogenic than the subclavian sites [12]. In our study, a thrombophlebitis on CVC was observed in five patients who had their CVC on the femoral site. We did not find a relationship between the formation of a thrombosis and the development of an infection related to the CVC. The femoral site seems to be the main factor favoring venous thrombosis in our series.

\section{Conclusion}

Non-infectious complications of non-tunneled central venous catheterization were frequent in our series. Some 
are potentially serious because that may, directly or not, be life-threatening. The main reason for these complications was the absence of ultrasound guidance due to insufficient equipment in our hemodialysis unit. Some aspects not less important as the deficiency in qualified human resources have contributed to this high frequency. Thus, our study reaffirms the need for an ultrasound-Doppler in hemodialysis units, even in developing countries, for a better patient safety and better legal coverage of the doctor performing the catheterization.

\section{Conflicts of interest}

The authors have no conflicts of interest to declare.

\section{Acknowledgements}

The authors thank Dr. Manan Hien for translating the text into English.

\section{References}

[1] Ethier, J., Mendelssohn, D.C., Elder, S.J., Hasegawa, T., Akizawa, T., Akiba, T., Canaud, B.J. and Pisoni, R.L. (2008) Vascular Access Use and Outcomes: An International Perspective for the Dialysis Outcomes and Practice Patterns Study. Nephrology Dialysis Transplantation, 23, 3219-3126. http://dx.doi.org/10.1093/ndt/gfn261

[2] Vats, H.S. (2012) Complications of Catheters: Tunneled and Nontunneled. Advances in Chronic Kidney Disease, 19, 188-194. http://dx.doi.org/10.1053/j.ackd.2012.04.004

[3] Kollo, D. (2013) Hyperkalemia in Chronic Hemodialysis Patients at the General Hospital of Yaoundé: Prevalence, Clinical and Electrocardiographic Aspects. Internal Medicine, University of Yaoundé, June, Accessed 1 July 2015, Available on the Website of Health Science and Diseases. http://www.hsd-fmsb.org/index.php/hsd/thesis/view/74

[4] Ka, E.H.F., Seck, M., Diakite, F., Cisse, M., Lemrabott, A.T., Faye, M., et al. (2014) Epidemiology of Cardiac and Vascular Calcification in Hemodialysis Patients in the CHU Aristide Le Dantec. 16th Joint Meeting of Society of Nephrology (SN) and the French Society of Dialysis (SFD). Nephrology \& Therapeutics, 10, 294-295. http://dx.doi.org/10.1016/j.nephro.2014.07.011

[5] Power, A., Singh, S., Ashby, D., Hamady, M., Moser, S., Gedroyc, W., Taube, D., Duncan, N. and Cairns, T. (2010) Translumbar Central Venous Catheters for Long-Term Hemodialysis. Nephrology Dialysis Transplantation, 25, 15881595. http://dx.doi.org/10.1093/ndt/gfp683

[6] (2012) Epidemiology and Information Network in Nephrology (REIN). Annual Report 2012, Agency of Biomedicine, Saint Denis La Plaine, France, Accessed on 2 April 2015, Available on the Website of the Agency of Biomedicine. http://www.agence-biomedecine.fr/

[7] (2014) United Nations Development Programme (UNDP). 2014 Report on Human Development, Ouagadougou, 17 October 2014.

[8] Ramilitiana, B., Rokotoarivony, S.T., Rabenjanahary, T., Razafimahefa, S.H., Soaniainamampionona, A.A., Randrianmarotia, W., et al. (2010) Epidemiological and Clinical Profile and Become of Chronic Renal Failure Hemodialysis Recipients in CHU HJRB Antananarivo Madagascar. Revue d'Anesthésie-Réanimation et de Médecine d'Urgence, 2, $11-14$.

[9] Centers for Disease Control and Prevention (2011) Vital Signs: Central Line Associated Blood Stream InfectionsUnited States, 2001, 2008, and 2009. Morbidity and Mortality Weekly Report, 60, 243-248.

[10] KDOQI (2006) Clinical Practice Guidelines and Clinical Practice Recommendations for 2006 Updates: Hemodialysis Adequacy, Peritoneal Dialysis Adequacy and Vascular Access. American Journal of Kidney Diseases, 48, 1-322.

[11] Sanogo, A. (2006) Study of Vascular Approaches in Hemodialysis in the Department of Nephrology of Hospital National du Point G. Medicine Thesis, Bamako, No. 90. http://www.keneya.net/fmpos/theses/2006/med/pdf/06M90.pdf

[12] McGee, D. and Gould, M. (2003) Preventing Complications of Central Venous Catheterization. The New England Journal of Medicine, 348, 1123-1133. http://dx.doi.org/10.1056/NEJMra011883

[13] Eisen, L.A., Narasimhan, M., Berger, J.S., Mayo, P.H., Rosen, M.J. and Schneider, M.F. (2006) Mechanical Complications of Central Venous Catheters. Intensive Care Medicine, 21, 40-46. http://dx.doi.org/10.1177/0885066605280884

[14] Merrer, J., Lefrant, J.Y. and Timsit, J.F. (2006) How to Optimize the Use of Central Venous Catheters in Intensive Care. Annales Françaises d'Anesthésie et de Réanimation, 25, 180-188. http://dx.doi.org/10.1016/j.annfar.2005.07.079

[15] Laksiri, L., dala-Fizelier, C. and Mimoz, O. (2008) Implementation of Central Venous Catheters: A Model of Quality 
Approach. Le Praticien en Anesthésie Réanimation, 12, 435-439.

[16] Iovino, F., Pittiruti, M., Buononato, M. and Lo Schiavo, F. (2001) Central Venous Access: Complications of the Different Routes of Central Approach. Annales de Chirurgie, 126, 1001-1006. http://dx.doi.org/10.1016/S0003-3944(01)00653-8

[17] Clark, E.G. and Barsuk, J.H. (2014) Temporary Hemodialysis Catheters: Recent Advances. Kidney International, 86, 888-895. http://dx.doi.org/10.1038/ki.2014.162

[18] Vinsonneau, C., Allain-Launay, E., Blayau, C., Darmon, M., Du Cheyron, D., Gaillot, T., et al. (2014) Extrarenal Purification in Adult and Pediatric Resuscitation. Formalized Recommendations of Experts under the Aegis of the French Language Resuscitation Society (FLRS), with the Participation of the French Society of Anesthesia and Resuscitation (FSAR), the Francophone Group of Resuscitation and Pediatric Emergencies (FGRPU) and Francophone Society of Dialysis (FSD), Resuscitation.

[19] Karakitsos, D., Labropoulos, N., De Groot, E., Patrianakos, A.P., Kouraklis, G., Poularas, J., Samonis, G., Tsoutsos, D.A., Konstadoulakis, M.M. and Karabinis, A. (2006) Real-Time Ultrasound-Guided Catheterization of the Internal Jugular Vein: A Prospective Comparison with the Landmark Technique in Critical Care Patients. Critical Care, 10, R162. http://dx.doi.org/10.1186/cc5101

[20] Mayoor, T.V., Juneja, D., Gopal, P.B., Sathyanarayanan, M., Subhramanyam, S., Gandhe, S., et al. (2010) UltrasoundGuided Femoral Dialysis Access Placement: A Single-Center Randomized. Clinical Journal of the American Society of Nephrology, 5, 235-239. http://dx.doi.org/10.2215/CJN.04920709

[21] Gallieni, M., Martina, V., Rizzo, M.A., Gravellone, L., Mobilia, F., Giordano, A., Cusi, D., et al. (2011) Central Venous Catheters: Legal Issues. The Journal of Vascular Access, 12, 273-279. http://dx.doi.org/10.5301/JVA.2011.7745

[22] Parienti, J.J., Megarbane, B. and Fischer, M.O. (2010) Catheter Dysfunction and Dialysis Performance According to Vascular Access among 736 Critically Ill Adults Requiring Renal Replacement Therapy: A Randomized Controlled Study. Critical Care Medicine, 38, 1118-1125.

[23] Kirkpatrick, W.G., Culpepper, R.M. and Sirmon, M.D. (1996) Frequency of Complications with Prolonged Femoral Vein Catheterization for Hemodialysis Access. Nephron, 73, 58-62.

[24] Timsit, J.F., Farkas, J.C., Boyer, J.M., Martin, J.B., Misset, B., Renaud, B., et al. (1998) Central Vein Catheter-Related Thrombosis in Intensive Care Patients: Incidence, Risks Factors, and Relationship with Catheter-Related Sepsis. Chest, 114, 207-213.

[25] Raad, II, Luna, M., Khalil, S.A., Costerton, J.W., Lam, C. and Bodey, G.P. (1994) The Relationship between the Thrombotic and Infectious Complications of Central Venous Catheters. Journal of the American Medical Association, 271, 1014-1016. 